\title{
Confined states and direction-dependent transmission in graphene quantum wells
}

\author{
J. Milton Pereira, Jr., V. Mlinar, and F. M. Peeters \\ Department of Physics, University of Antwerp, Groenenborgerlaan 171, B-2020 Antwerpen, Belgium \\ P. Vasilopoulos \\ Department of Physics, Concordia University, Montreal, Quebec, Canada H3G 1M8 \\ (Received 24 April 2006; published 24 July 2006)
}

\begin{abstract}
We report the existence of confined massless fermion states in a graphene quantum well $(\mathrm{QW})$ by means of analytical and numerical calculations. These states show an unusual quasilinear dependence on the momentum parallel to the QW: their number depends on the wave vector and is constrained by electron-hole conversion in the barrier regions. An essential difference with nonrelativistic electron states is a mixing between free and confined states at the edges of the free-particle continua, demonstrated by the direction-dependent resonant transmission across a potential well.
\end{abstract}

DOI: 10.1103/PhysRevB.74.045424

PACS number(s): 73.21.-b, 71.10.Pm, 81.05.Uw

Recent studies have demonstrated the production of stable, ultrapure, two-dimensional (2D) carbon crystals, also known as graphene. ${ }^{1-3}$ These $2 \mathrm{D}$ crystals possess unusual properties, such as unconventional quantum Hall effect ${ }^{4-7}$ and a strong electric-field effect. ${ }^{8}$ A large part of these new properties is a consequence of the linear (in wave vector) energy spectrum near the Fermi energy and are expected to lead to a new class of carbon- or graphene-based nanoelectronic devices. Previous theoretical studies of relativistic fermions interacting with strong fields have indicated that the quantum behavior of the particles may differ considerably from the nonrelativistic case. ${ }^{9}$ In this paper, we investigate the nature of electron states in graphene QWs and their quantized spectrum.

Graphene layers consist of a honeycomb lattice of covalent-bond carbon atoms, which can be treated as two interpenetrating triangular sublattices, labeled $A$ and $B$, and are often discussed in terms of unrolled, single-wall carbon nanotubes. The low-energy band structure of graphene is gapless and the corresponding electronic states are found near two cones located at unequivalent corners of the Brillouin zone. ${ }^{10}$ The low-energy carrier dynamics is equivalent to that of a $2 \mathrm{D}$ gas of massless charged fermions. Their behavior is governed by the 2D Dirac Hamiltonian, ${ }^{11,12}$

$$
\mathcal{H}=v_{F}(\vec{\sigma} \cdot \hat{\mathbf{p}})
$$

where the pseudospin matrix $\vec{\sigma}$ has components given by Pauli's matrices, $\hat{\mathbf{p}}=\left(p_{x}, p_{y}\right)$ is the momentum operator, and $v_{F}$ is the effective speed of light of the system, which in this case corresponds to the Fermi velocity $v_{F} \approx 1 \times 10^{6} \mathrm{~m} / \mathrm{s}$. The Hamiltonian (1) acts on the states represented by the two-component spinors $\Psi=\left[\psi_{A}, \psi_{B}\right]^{T}$, where $\psi_{A}$ and $\psi_{B}$ represent the envelope functions associated with the probability amplitudes at the respective sublattice sites of the honeycomb graphene structure. The low-energy spectrum of free carriers is $E= \pm \hbar v_{F}\left(k_{x}^{2}+k_{y}^{2}\right)^{1 / 2}$, with $k_{x}$ and $k_{y}$ the wave vector along the $x$ and $y$ axes, in the vicinity of the cones at the Brillouin zone; the $+(-)$ sign refers to electron (hole) bands. Equation (1) also implies that the carriers are chiral particles, with the pseudospin aligned parallel (antiparallel) to the direction of propagation of the electrons (holes).

Representing the effect of an external electrostatic field by an external potential $U$ and including a diagonal effective mass-like term $m v_{F}^{2}$ leads to the Dirac equation

$$
\left[v_{F}(\vec{\sigma} \cdot \hat{\mathbf{p}})+m v_{F}^{2} \sigma_{z}\right] \Psi=(E-U) \Psi
$$

The term $\propto m v_{F}^{2}$ creates a gap in the dispersion and may arise from spin-orbit interaction or from the coupling between the graphene layer and the substrate. ${ }^{13}$ For a circularly symmetric potential with $m=0$, the solutions inside the potential well match free-particle solutions outside, therefore ruling out bound states. ${ }^{14}$ This is caused by the conservation of the chirality in the interaction with the potential and the absence of a gap in the spectrum and can be understood as a manifestation of a relativistic tunneling effect first discussed by Klein ${ }^{15,16}$ for one-dimensional (1D) potentials, in which Dirac fermions can propagate to hole states across a steep potential barrier without any damping. For massless particles this tunneling is expected to occur for any value of $U_{0}$. However, as we show below, for a $1 \mathrm{D}$ potential a finite value of the momentum parallel to the potential barrier can suppress this tunneling and thus allow the confinement of electrons. Very recent studies have demonstrated the confinement of electrons in a graphene strip. ${ }^{17}$ In this case, in order to obtain the confinement the authors assumed a position-dependent effective mass for the particles. This assumption does not permit the observation of Klein tunneling and of the momentum-dependent reflection and transmission. Therefore, the confinement in this case is qualitatively different from ours specified below. In order to demonstrate the confinement in an electrostatic quantum well, we consider a zero or constant effective mass throughout the system and first a $1 \mathrm{D}$ square-well potential $U(x)=U_{0} \theta(|x|-L / 2), U_{0}>0$, cf. Fig. 1, which allows an analytical solution for the eigenstates and sheds light on some general features of the problem. Later on, we consider a parabolic confinement.

With momentum conservation in the $y$ direction, we look for solutions in the form $\psi_{C}(x, y)=\phi_{C}(x) e^{i k_{y} y}, C=A, B$, and obtain

$$
d \phi_{B} / d \xi+\beta \phi_{B}=i(\epsilon-u-\Delta) \phi_{A},
$$




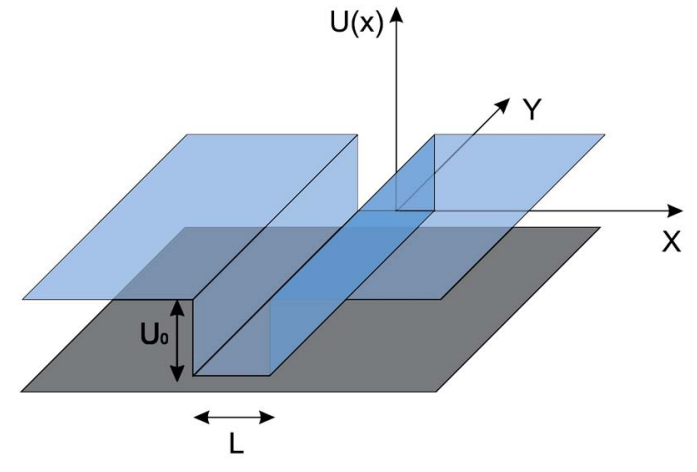
layer.

FIG. 1. (Color online) A square quantum well on a graphene

$$
d \phi_{A} / d \xi-\beta \phi_{A}=i(\epsilon-u+\Delta) \phi_{B},
$$

where $\xi=x / L, \quad \beta=k_{y} L, \quad \epsilon=E L / \hbar v_{F}, \quad u=U(x) L / \hbar v_{F}$, and $\Delta=m v_{F} L / \hbar$ (for graphene $\hbar v_{F}=0.539 \mathrm{eV} \mathrm{nm}$ ). Decoupling Eqs. (3) and (4) gives for $\phi_{A}$ the result

$$
\begin{aligned}
\frac{d^{2} \phi_{A}}{d \xi^{2}} & +\frac{u^{\prime}}{(\epsilon-u+\Delta)} \frac{d \phi_{A}}{d \xi} \\
- & {\left[\beta^{2}+\beta \frac{u^{\prime}}{(\epsilon-u+\Delta)}-(\epsilon-u)^{2}+\Delta^{2}\right] \phi_{A}=0, }
\end{aligned}
$$

where $u^{\prime}$ is the derivative of the potential. For a square well, these derivatives are Dirac $\delta$ functions.

The character of the solutions depends on the value of $\beta$, which determines the sign of the last term on the left side of Eq. (5). The solutions are of three types: (i) traveling waves, which describe free electrons, free holes, as well as mixed states that occur due to the Klein tunneling of electrons to holes outside the potential well; (ii) standing waves, which for massless fermions arise only from finite values of $\beta$ above an energy-dependent cutoff and decay exponentially in the barrier regions; and (iii) tunneling waves, which are oscillatory outside the well whereas inside it they are combinations of exponentials with real exponents; these correspond to holes that undergo ordinary tunneling across the potential well. Type (ii) solutions occur in energy and wavevector ranges for which there are no hole states available at the barrier regions. This suppresses the Klein tunneling, since it depends on the electron-hole conversion at the interface.

In this work, we focus on type (ii) solutions which describe electron states confined across the well and propagating along it. Their energies are in the region delimited by the curves $E=\left[\left(\hbar v_{F} k_{y}\right)^{2}+m^{2} v_{F}^{4}\right]^{1 / 2}+U_{0}$ and $E=\left[\left(\hbar v_{F} k_{y}\right)^{2}+m^{2} v_{F}^{4}\right]^{1 / 2}$. At smaller wave vectors, tunneling across the barriers introduces a cutoff in the spectrum for $E<-\left[\left(\hbar v_{F} k_{y}\right)^{2}+m^{2} v_{F}^{4}\right]^{1 / 2}+U_{0}$. For confined states, the spinor components decay exponentially in the region $\xi<-1 / 2$. Then the $A$ component can be written as $\phi_{A}(\xi)=A_{1} e^{\alpha \xi}$. Substituting $\phi_{A}(\xi)$ in Eq. (4) we find $\phi_{B}(\xi)=i f \_A_{1} e^{\alpha \xi}$, with $f_{-}=(\beta-\alpha) /\left(\epsilon-u_{0}+\Delta\right)$ and the decay constant $\alpha$ given by $\alpha=\left[\beta^{2}-\left(\epsilon-u_{0}\right)^{2}+\Delta^{2}\right]^{1 / 2}$, where $u_{0}=U_{0} L / \hbar v_{F}$.

The solutions $\phi_{A}$ and $\phi_{B}$ for $|\xi| \leqslant 1 / 2$ are of the type

$$
\begin{gathered}
\phi_{A}(\xi)=C_{2} \cos (\kappa \xi)+D_{2} \sin (\kappa \xi), \\
\phi_{B}(\xi)=[i /(\epsilon+\Delta)]\left\{C_{2}[\beta \cos (\kappa \xi)+\kappa \sin (\kappa \xi)]\right. \\
\left.+D_{2}[\beta \sin (\kappa \xi)-\kappa \cos (\kappa \xi)]\right\},
\end{gathered}
$$

with $\kappa^{2}=\epsilon^{2}-\beta^{2}-\Delta^{2}$. For $\xi>1 / 2$, the solutions are similar to those for $\xi<-1 / 2$ but with a negative exponent: $\phi_{A}(\xi)$ $=A_{3} e^{-\alpha \xi}, \phi_{B}(\xi)=i f_{+} A_{3} e^{-\alpha \xi}$, where $f_{+}=(\beta+\alpha) /\left(\epsilon-u_{0}+\Delta\right)$. It should be stressed that, in contrast with the nonrelativistic case, the spinor components are neither even nor odd functions, despite the symmetry of the potential. This symmetry, however, is reflected in the probability density $\rho=\Psi^{\dagger} \Psi$ $=\phi_{A}(\xi)^{\dagger} \phi_{A}(\xi)+\phi_{B}(\xi)^{\dagger} \phi_{B}(\xi),{ }^{14}$ which is an even function. Moreover, for a step potential the derivatives of the spinor components are not continuous because $u^{\prime}$ in Eq. (5) is a $\delta$ function. This can be demonstrated by considering the continuity of the $y$ component of the probability current, $j_{y}=v_{f} \Psi^{\dagger} \sigma_{y} \Psi$, across the potential interface: using Eqs. (3) and (4), we obtain $\left[u_{+}=u_{0} /(\epsilon+\Delta)\right]$

$$
\phi_{A \leftarrow}^{\prime}(1 / 2)=\left(1-u_{+}\right) \phi_{A \rightarrow}^{\prime}(1 / 2)+u_{+} \beta \phi_{A}(1 / 2),
$$

where the arrows indicate the limiting values from the left and right of the interface. Notice in Eq. (8) that, even for large values of $\Delta$, a continuous derivative of $\phi_{A}$ may be assumed only for $u_{0} \beta \ll \Delta$.

Requiring the continuity of $\phi_{A}$ and $\phi_{B}$ at $\xi=-1 / 2$ and $1 / 2$, we obtain the following transcendental equation for the energy eigenvalues:

$$
S_{-}(\epsilon, \beta,+1) S_{+}(\epsilon, \beta,+1)+S_{-}(\epsilon, \beta,-1) S_{+}(\epsilon, \beta,-1)=0,
$$

where $S_{ \pm}(\epsilon, \beta, s)=\beta-f_{ \pm}(\epsilon+\Delta)-s \kappa \delta^{\overline{+} s}$ and $\delta=\tan (\kappa / 2)$. The nonrelativistic limit can be obtained using $\epsilon=\epsilon_{c}+\Delta$, where $\epsilon_{c}$ corresponds to the classical energy and considering the limit $\Delta \gg \epsilon_{c}$, to give

$$
f_{ \pm}(\epsilon+\Delta) \approx(\beta \pm \alpha)(1+\Gamma),
$$

where $\alpha \approx\left[\beta^{2}+2 \Delta\left(u_{0}-\epsilon_{c}\right)\right]^{1 / 2}, \quad \kappa \approx\left(2 \Delta \epsilon_{c}-\beta^{2}\right)^{1 / 2}$, and $\Gamma$ $\equiv u_{0} / 2 \Delta$. Equation (9) then becomes $(\bar{\kappa}=\kappa / 2)$

$$
[\alpha(1+\Gamma)-\kappa \tan \bar{\kappa}][\alpha(1+\Gamma)+\kappa \cot \bar{\kappa}]-2(\beta \Gamma)^{2}=0 .
$$

For $\Gamma \ll 1$ and $\beta u_{0} \ll \Delta$, we recover the familiar transcendental equation for a nonrelativistic QW. In this limit, a nonzero value for $\beta$ is equivalent to a simple shift of the energy scale $\epsilon^{\prime} \rightarrow \epsilon_{c}-\beta^{2} / 2 \Delta$ and the spectrum of the confined states becomes a set of nested parabolas. On the other hand, Eq. (11) shows that, even for massive particles, the QW spectrum does depend on the $y$ component of the momentum, in contrast with the non-relativistic results. Thus, a significant modification of the parabolic spectrum occurs as $\beta$ increases.

Equation (9) was solved numerically. The results are shown in Fig. 2 for $U_{0}=50 \mathrm{meV}, L=200 \mathrm{~nm}$, and $\Delta=0$. The dashed lines delimit the continuum region, which corresponds to free electrons $\left(E \geqslant \hbar k_{y}+U_{0}\right)$ with energies greater than the barrier height, and free holes $\left(E \leqslant-\hbar v_{F} k_{y}+U_{0}\right)$ that propagate in the system by means of the Klein tunneling 


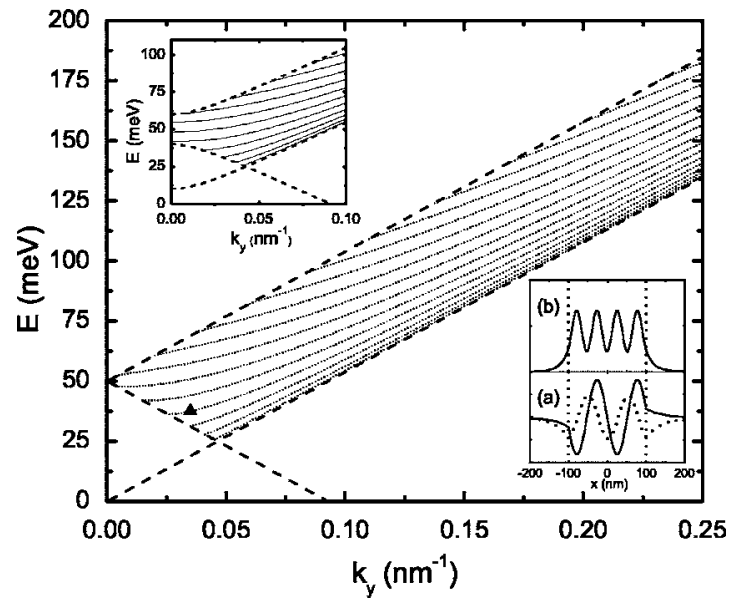

FIG. 2. Spectrum of confined states in a graphene square QW vs $k_{y}$ for $U_{0}=50 \mathrm{meV}, L=200 \mathrm{~nm}$, and $m v_{F}^{2}=0$. The lower inset shows (a) $\phi_{A}$ and $i \phi_{B}$ for the state shown by the solid triangle and (b) the related probability density. The upper inset shows the effect of a nonzero mass, for $m v_{F}^{2}=10 \mathrm{meV}$.

mechanism. The cutoff at low wave vectors thus arises due to the conversion of confined electrons to free holes. For large values of $k_{y}$, the dispersion branches are given approximately by

$$
E=\hbar v_{F}\left[(n \pi / L)^{2}+k_{y}^{2}\right]^{1 / 2},
$$

where $n$ is an integer. For any given $k_{y}$, the accuracy of this approximation improves as $L$ increases. The lower inset shows (a) $\phi_{A}$ (solid curve) and $i \phi_{B}$ (dashed curve) for the confined state, with $k_{y}=0.03 \mathrm{~nm}^{-1}$, shown by the solid triangle and (b) the corresponding probability density in arbitrary units. The plot clearly indicates a discontinuity in the derivative of the spinor component functions at the barrier interfaces. The vertical dotted lines indicate the walls of the well. The upper inset shows the effect of the mass, with $m v_{F}^{2}=10 \mathrm{meV}$. The dashed lines again represent the limits of the free-particle continua. In this case, confined states are allowed, for $k_{y}=0$, in the range $u_{0}-\Delta<\epsilon<u_{0}+\Delta$. This energy range broadens as $k_{y}$ increases and remains constant for $k_{y}>\left(u_{0}^{2} / 4-\Delta^{2}\right)^{1 / 2}$. At lower energies, there is again a cutoff, due to the Klein tunneling at the barriers, which disappears for $2 \Delta>u_{0}$.

Next, we consider a $\mathrm{QW}$ with a parabolic potential profile $U(x)=U_{0}(2 x / L)^{2}$ for $|x| \leqslant L / 2$ and $U(x)=U_{0}$ for $|x|>L / 2$. Figure 3 shows the spectrum of confined states obtained from a numerical solution of Eqs. (3) and (4) for $U_{0}=50 \mathrm{meV}$ and $L=200 \mathrm{~nm}$. The results are qualitatively similar to those of the previous case, but now with the eigenvalues being approximately equally spaced for large wave vectors.

An essential difference with nonrelativistic electrons, evident in all cases, is the appearance of new confined states at the edges of the continua, where the quantized electron branches intercept the free-particle regions. Thus, by an adiabatic increase in $k_{y}$ one can transform a free-electron or a free-hole state into a bound electron state. This occurs because the presence of the barriers allows a mixing of electron

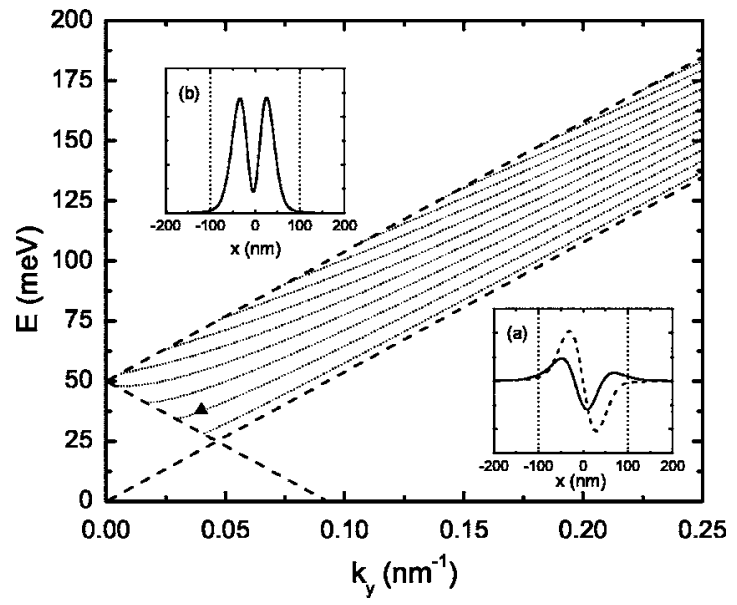

FIG. 3. As in Fig. 2 but for a parabolic QW.

and hole states with the same energy and $y$ component of momentum. As a result there is constructive interference between confined states and unbound electron or hole states that are resonantly transmitted across the QW. We demonstrate this by calculating the transmission coefficient of electrons incident on a square well. Consider the propagating solutions $\psi_{A}(x, y)=\phi_{A}(x) e^{i k_{y} y}$, with

$$
\phi_{A}(x)=\left\{\begin{array}{cc}
e^{i \alpha \xi}+B_{1} e^{-i \alpha \xi}, & \xi<-1 / 2, \\
A_{2} e^{i \kappa \xi}+B_{2} e^{-i \kappa \xi}, & -1 / 2 \leqslant \xi \leqslant 1 / 2, \\
A_{3} e^{i \alpha \xi}, & \xi>1 / 2,
\end{array}\right.
$$

where $\alpha=\left[\left(\epsilon-u_{0}\right)^{2}-\beta^{2}-\Delta^{2}\right]^{1 / 2}$; the solutions for $\phi_{B}$ are obtained as in the previous calculation. Then, the transmission coefficient is obtained as $T=\left|A_{3}\right|^{2}$, where

$$
A_{3}=\frac{\left(g_{+}-g_{-}\right)\left(f_{+}-f_{-}\right) e^{-i \alpha}}{\left(g_{+}-f_{+}\right)\left(g_{-}-f_{-}\right) e^{2 i \kappa}-\left(g_{+}-f_{-}\right)\left(g_{-}-f_{+}\right)},
$$

$g_{ \pm}=(\beta \pm i \alpha) /(\epsilon+\Delta)$ and $f_{ \pm}=(\beta \pm i \kappa) /\left(\epsilon-u_{0}+\Delta\right)$. A $\left(k_{y}, \alpha / L\right)$ contour plot of the transmission $T$ is shown in Fig. 4 for $U_{0}=50 \mathrm{meV}$ and $L=200 \mathrm{~nm}$. As seen, $T$ depends on the di-

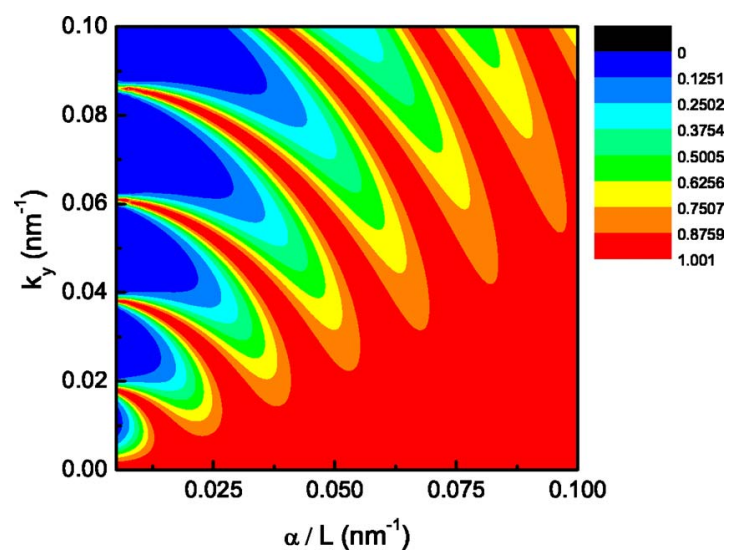

FIG. 4. (Color online) Contour plot of the transmission coefficient of electrons incident on a graphene square well, with energy $E>U_{0}$, as a function of $\alpha$, for $U_{0}=50 \mathrm{meV}, L=200 \mathrm{~nm}$, and $m=0$. 
rection of propagation and displays an oscillatory behavior. As $\alpha \rightarrow 0, T$ reaches a series of maxima for values of $\beta$ that coincide with the wavevectors for which mixing occurs, cf. Fig. 2. Notice that for a significant range of incident angles $T$ is always equal to 1 . This includes the case of nearly normal incidence, $k_{y} \approx 0$, and is in sharp contrast with the nonrelativistic case in which $T$ exhibits periodic maxima equal to 1 as a function of $k_{x}$. A similar direction-dependent transmission through graphene barriers was reported recently. ${ }^{18} \mathrm{~A}$ direction-dependent transmission is also possible for nonrelativistic electrons tunneling through magnetic barriers. ${ }^{19}$

The $y$ components of the momentum for which mixing is allowed correspond to confined states for which the asymptotic limit $\alpha \rightarrow 0$ applies. This yields the condition $\sin (\kappa)=0$ or $\kappa=n \pi$, where $n$ is an integer. Using the definition of $\kappa$ and $\alpha$ gives

$$
\beta=\left[\left(\frac{n^{2} \pi^{2}}{2 u_{0}}-\frac{u_{0}}{2}\right)^{2}-\Delta^{2}\right]^{1 / 2} .
$$

Since $\beta^{2}>0$, the values of $n$ can be obtained from the condition $\pm\left(n^{2} \pi^{2} / 2 u_{0}-u_{0} / 2\right) \geqslant \Delta$, where the $+(-)$ sign is associated with the upper (lower) continuum edges. From this condition we find that for $U_{0}<2 m v_{F}^{2}$ there is no mixing at lower energies, although it persists at the upper continuum edge and the minimum value of $\beta$ for the mixing increases with $\Delta$.

A complementary way to see the direction dependence of the transmission $T$ is shown in Fig. 5(a), with $T$ plotted versus the angle of incidence $\theta=\arctan \left(k_{y} / \alpha\right)$, for different electron energies as indicated. The QW parameters are $L=200$ and $U_{0}=50 \mathrm{meV}$. Notice that for $\theta \approx 0$, we have $T \approx 1$ in agreement with the $k_{y} \approx 0$ part of Fig. 4. In Fig. 5(b), we plot $T$ versus the energy $E$ for $\theta=\pi / 3$. As seen, $T$ oscillates with the energy due to the resonance effect caused by the confined states (as in the Ramsauer-Townsend effect). The energies for the maxima of the transmission can be obtained from Eq. (15) as $\epsilon=(n \pi)^{2} / 2 u_{0}+u_{0} / 2$.

In summary, we showed that it is possible to confine massless charge carriers by means of electrostatic potentials, due to the wave-vector-dependent suppression of the
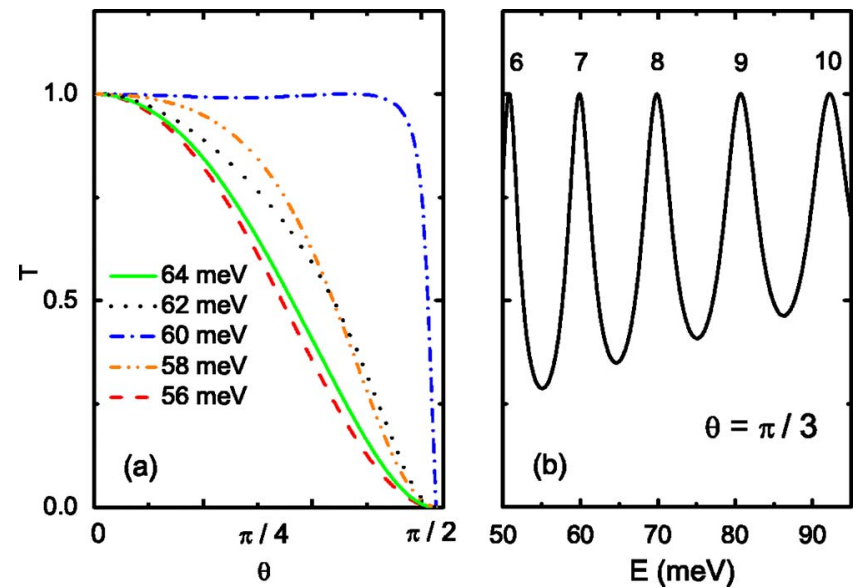

FIG. 5. (Color online) (a) Transmission $T$ vs angle of incidence $\theta$ for different energies as indicated. (b) $T$ vs energy $\mathrm{E}$ for $\theta=\pi / 3$. The maxima are marked by the numbers of the confined states.

electron-hole conversion at the potential barriers. We thus obtained the quantized spectrum of confined electron states in graphene quantum wells as a function of the $y$ component of the wave vector. The results show a remarkable dependence of the eigenvalues on the momentum with a cutoff at low wave vectors. The relativistic correction to the classical QW spectrum leads to a wave-vector dependence of the number of confined states due to the electron-hole conversion at the continuum edges. Accordingly, such QWs must be treated as inherently 2D systems. This is further demonstrated by the directional dependence of the transmission shown in Figs. 4 and 5. Studying the resonance transmission of electrons across a QW with energies above the height of the confining walls, $E>U_{0}$, can probe the discrete levels which can be populated by tuning the Fermi energy of the system with the electric-field effect. ${ }^{1}$

This work was supported by the Brazilian Council for Research $(\mathrm{CNPq})$, the Flemish Science Foundation (FWOVl), the Belgian Science Policy (IUAP), and the Candian NSERC Grant No. OGP0121756.
${ }^{1}$ K. S. Novoselov, A. K. Geim, S. V. Morozov, D. Jiang, Y. Zhang, S. V. Dubonos, I. V. Grigorieva, and A. A. Firsov, Science 306, 666 (2004).

${ }^{2}$ K. S. Novoselov, D. Jiang, F. Schedin, T. J. Booth, V. V. Khotkevich, S. V. Morozov, and A. K. Geim, Proc. Natl. Acad. Sci. U.S.A. 102, 10451 (2005).

${ }^{3}$ Y. Zhang, J. P. Small, W. V. Pontius, and P. Kim, Appl. Phys. Lett. 86, 073104 (2005).

${ }^{4}$ Y. Zheng and T. Ando, Phys. Rev. B 65, 245420 (2002).

${ }^{5}$ V. P. Gusynin and S. G. Sharapov, Phys. Rev. Lett. 95, 146801 (2005).

${ }^{6}$ K. S. Novoselov, A. K. Geim, S. V. Morozov, D. Jiang, M. I. Katsnelson, I. V. Grigorieva, S. V. Dubonos, and A. A. Firsov, Nature (London) 438, 197 (2005).
${ }^{7}$ Y. Zhang, Y. W. Tan, H. L. Stormer, and P. Kim, Nature (London) 438, 201 (2005)

${ }^{8}$ Y. Zhang, J. P. Small, M. E. S. Amori, and P. Kim, Phys. Rev. Lett. 94, 176803 (2005).

${ }^{9}$ J. Reinhardt and W. Greiner, Rep. Prog. Phys. 40, 219 (1977); V. Petrillo and Davide Janner, Phys. Rev. A 67, 012110 (2003).

${ }^{10}$ P. R. Wallace, Phys. Rev. 71, 622 (1947); M. Wilson, Phys. Today 59(1), 21 (2006).

${ }^{11}$ G. W. Semenoff, Phys. Rev. Lett. 53, 2449 (1984).

${ }^{12}$ I. A. Luk'yanchuk and Y. Kopelevich, Phys. Rev. Lett. 93, 166402 (2004).

${ }^{13}$ C. L. Kane and E. J. Mele, Phys. Rev. Lett. 95, 226801 (2005).

${ }^{14}$ D. P. DiVincenzo and E. J. Mele, Phys. Rev. B 29, 1685 (1984).

${ }^{15}$ O. Klein, Z. Phys. 53, 157 (1929). 
${ }^{16}$ N. Dombey and A. Calogeracos, Phys. Rep. 315, 41 (1999).

${ }^{17}$ N. M. R. Peres, A. H. Castro Neto, and F. Guinea, cond-mat/ 0603771 (unpublished); J. Tworzydło, B. Trauzettel, M. Titov, A. Rycerz, and C. W. J. Beenakker, Phys. Rev. Lett. 96, 246802 (2006)
${ }^{18}$ M. I. Katsnelson, K. S. Novoselov, and A. K. Geim, cond-mat/ 0604323 (unpublished); V. V. Cheianov and V. Falko, cond-mat/ 0603624 (unpublished).

${ }^{19}$ A. Matulis, F. M. Peeters, and P. Vasilopoulos, Phys. Rev. Lett. 72, 1518 (1994). 